\title{
Patient with Chronic Myeloid Leukemia: A Case Study
}

Muhammad Zeeshan Zafar*

Department of Pharmacy, University of Sargodha, Pakistan

\begin{abstract}
Introduction: Chronic myeloid leukemia abbreviated as CML is a type of myeloproliferative disease characterized by the increased and unregulated growth of predominantly myeloid cells in the bone marrow and the accumulation of these cells in the blood. It is a clonal bone marrow stem cell disorder in which a proliferation of mature granulocytes and their precursor is found.
\end{abstract}

Case presentation: We report the case of 58-year-old Asian lady experiencing generalized body weakness, pallor, left upper abdominal pain and sudden weight loss of $5 \mathrm{~kg}$ within three weeks and a high blood pressure of 190/110. She has initially diagnosed splenomegaly and a mild heart attack. Various laboratory tests including complete blood count were conducted and the test results revealed a myeloproliferative disorder. The oncologist suggested bone marrow aspiration that led to a diagnosis of chronic myeloid leukemia. The disease was thought treated at an early stage but the case became complicated by previous untreated pathological conditions.

Conclusion: Not all cases of CML are complicated in such a critical manner. Our report emphasizes on the fact of the need of good clinical evaluation by a qualified therapist and use of appropriate investigative studies is mandatory in order to secure patient from such a critical health condition.

Keywords: Chronic myeloid leukemia; Medical care

\section{Introduction}

Myeloid leukemia (AML) is a form of cancer that is characterized by infiltration of the bone marrow, blood, and other tissues by proliferative, clonal, abnormally differentiated, and occasionally poorly differentiated cells of the hematopoietic system. Chronic myeloid leukemia abbreviated as CML is a cancer of white blood cells. It is a form of leukemia characterized by the increased and unregulated growth of predominantly myeloid cells in the bone marrow and the accumulation of these cells in the blood [1]. It is a clonal bone marrow cell disorder in which there is a proliferation of mature granulocytes (neutrophils, eosinophil, and basophils) and their precursor associated with a characteristic chromosomal translocation called the Philadelphia chromosome [2].

In Western countries, it accounts for $15 \%$ to $20 \%$ of all adult leukemia and $14 \%$ of leukemia overall including pediatric population. Incidence rate varies from 0.6 to 2.0 cases per 100,000 inhabitants and is higher in men than in women (male to female ratio of 1:4:1). Geographic and/or ethnic variation might contribute to the variability of incidences among the registries. In most cases, no obvious cause for CML can be isolated. It appears more commonly in elderly with a median age at diagnosis of 65 years [1].

Radiation exposure can increase the risk of developing CML but it takes several years to develop leukemia from radiation exposure. Most people treated for cancer do not develop leukemia and also most patients with CML have not been exposed to radiation.

\section{Case Presentation}

A 58-years-old, 68-Kg Asian lady was presented to local hospital, Lahore Cantt. Pakistan. She was experiencing generalized body weakness, pallor, left upper abdominal pain and a rapid weight loss of 5-Kgs within three weeks [3]. The vital symptoms showed blood pressure (BP) 190/110 mmHg; Temperature $98^{\circ} \mathrm{F}$; Pulse rate 82 beats/ minute and splenomegaly. She was a housewife and used to live in an environment with no known exposure to chemicals, fumes, radiations and other environmental allergens. Laboratory tests including complete blood count, liver function test, blood urea nitrogen test, serum uric acid, serum triglyceride test and blood sugar test came out to be significantly abnormal, complicating the case. From complete blood count, the hematologist diagnosed myeloid-proliferative disorder and patient was recommended to have bone-marrow aspirate and Philadelphia chromosome or BCR-ABL1 fusion gene analysis by fluorescent in situ hybridization (FISH) technic. Bone marrow aspirate and the positive result for Philadelphia chromosome test led to the diagnosis of CML [4-6].

On the basis of medical investigation Physician prescribed oneweek therapy including Hydra (Hydroxyurea) $500 \mathrm{mg}$ capsule (Oral) T.D (twice a day); Zyloric (Allopurinol) $300 \mathrm{mg}$ tablets (Oral) O.D (once a day); Norvasc (Amlodipine besylate) $10 \mathrm{mg}$ tablets (Oral) O.D; Lipitor (Atorvastatin) $10 \mathrm{mg}$ tablets (Oral) O.D; Pozen (Pioglitazone) $15 \mathrm{mg}$ tablets (Oral) T.D; Methylcobal (Vitamin B-12) $100 \mathrm{mcgs}$ injection (Intra-muscular) once a week. In the meanwhile, patient experienced severe side effects such as swelling of lips, tongue, and throat, swelling of hands and feet, muscular pain. Uncontrolled blood pressure and radiating sudden chest pain further worsen the patient's condition. So, the patient had a revisit to the doctor within three days of recommended therapy. On revisit, the Oncologist made few changes in the treatment regime. Tablet lipitor and tablet piozen were excluded and Amaryl (Glimepiride) $2 \mathrm{mg}$ tablet (Oral) once a day; Phlogin (Diclofenac sodium) $75 \mathrm{mg} / 3 \mathrm{ml}$ ampoule injection (Intramuscular) once a day were added. On revisit, after one week of therapy, different laboratory tests of a patient were conducted. CBC test came out to be

*Corresponding author: Muhammad Zeeshan Zafar, Department of Pharmacy, University of Sargodha, Pakistan, Tel: 03466189496; E-mail: shanmughal11@gmail.com

Received August 13, 2017; Accepted September 13, 2017; Published September 18, 2017

Citation: Zafar MZ (2017) Patient with Chronic Myeloid Leukemia: A Case Study. J Cancer Sci Ther 9: 635-636. doi:10.4172/1948-5956.1000485

Copyright: (c) 2017 Zafar MZ. This is an open-access article distributed under the terms of the Creative Commons Attribution License, which permits unrestricted use, distribution, and reproduction in any medium, provided the original author and source are credited. 
normal. Oncologist demonstrated that the disease was treated but not cured. So, the patient was suggested to have a bone marrow biopsy and other laboratory tests every month as a prophylaxis against recurrence.

\section{Discussion}

This is a case study of CML in an elderly patient that was complicated by untreated coronary artery disease. Dosage regime designed while ignoring patient's critical medical history further complicated patient's condition. Side effects of medication led oncologist to design a new treatment plan that led to treatment but not cure of disease. Angioplasty should be recommended that was not.

British national formulary is one of the standard books used to design the treatment plan. CML can be treated by chemotherapy. Busulfan was the first drug to be effective in producing hematologic remission. Hydroxyurea is equally effective but less mutagenic and has more favorable toxicity profile than Busulfan. Interferon- $\alpha$ can produce hematologic remission and can eliminate Philadelphia positive clone when assessed by classical cytogenetics. This drug is also used in combination with Cytarabine. Hematopoietic Cell Transplantation is treatment employed following high dose chemotherapy and can result in long term remission and a possible cure. However, the number of patients who can benefit from this method is limited by the availability of suitable donors and age limits [7]. The recent introduction of STI 571 (Signal transduction inhibitor) has been widely reported and has gained considerable public attention. This molecule binds to the bar/abl protein and results in prevention of phosphorylation of any substrate. This drug has a high potency with a mild toxicity profile and is administered.

Usmani SZ has performed retrospective study in Shaukat Khanum hospital Pakistan and collected 461 samples with myeloid leukemia and proposed that most patients were affected with this disease at their adult age.

Busulfan was the first drug to be effective in producing hematologic remission. Hydroxyurea is equally effective but less mutagenic and has more favorable toxicity profile than Busulfan. Interferon-a can produce hematologic remission and can eliminate Philadelphia positive clone when assessed by classic cytogenetic. This drug is also used in combination with Cytarabine [8].

\section{Conclusion}

Not all cases of chronic myeloid leukemia are complicated in such a critical manner. Our report emphasizes on the fact of the need of good clinical evaluation by a qualified therapist and use of appropriate investigative studies is mandatory in order to secure patient from such a critical health condition. This case report was as complicated as you seen above disease was diagnosed after so many laboratory tests was performing.

\section{References}

1. Döhner H, Weisdorf DJ, Bloomfield CD (2015) Acute myeloid leukemia. N Engl J Med 373: 1136-1152.

2. Kantarjian H, Cortes J (2013) Chronic myeloid leukemia. Niederhuber JE, Armitage JO, Dorohow JH (eds). Abeloff's Clinical Oncology. (5th edn). Philadelphia, USA.

3. Anastai J, Vardiman JW (2000) Chronic myelogenous leukemia and the chronic myeloproliferative diseases. In: Knowles DM. Neoplastic Hematopathology (2nd edn). pp. 1745-1760.

4. Burnett A (2013) AML 12-Acute myeloid leukemia Adults. University of Wales College of Medicine, UK.

5. Döhner K, Paschka P, Döhner H (2015) Acute Myeloid Leukaemia Internist (Berl) 56 354-363.

6. Zeeshan Zafar M, Asim M, Ahsan M, Bashir T, Rasheed S (2017) A case report Typhoid resistant to chloramphenicol. J Integr Oncol 6: 189.

7. Brunning D, Mckenna RW (1993) Chronic myeloid leukemia. In: Atlas of tumor pathology. Tumors of bone marrow. 3rd Series. pp. 195-222.

8. Goldman J, Melo JV (2003) Chronic myeloid dilemma. European leukemia advances in biology and new approaches to treatment. $\mathrm{N}$ Engl $\mathrm{J}$ Med 349: 1451-1464. 